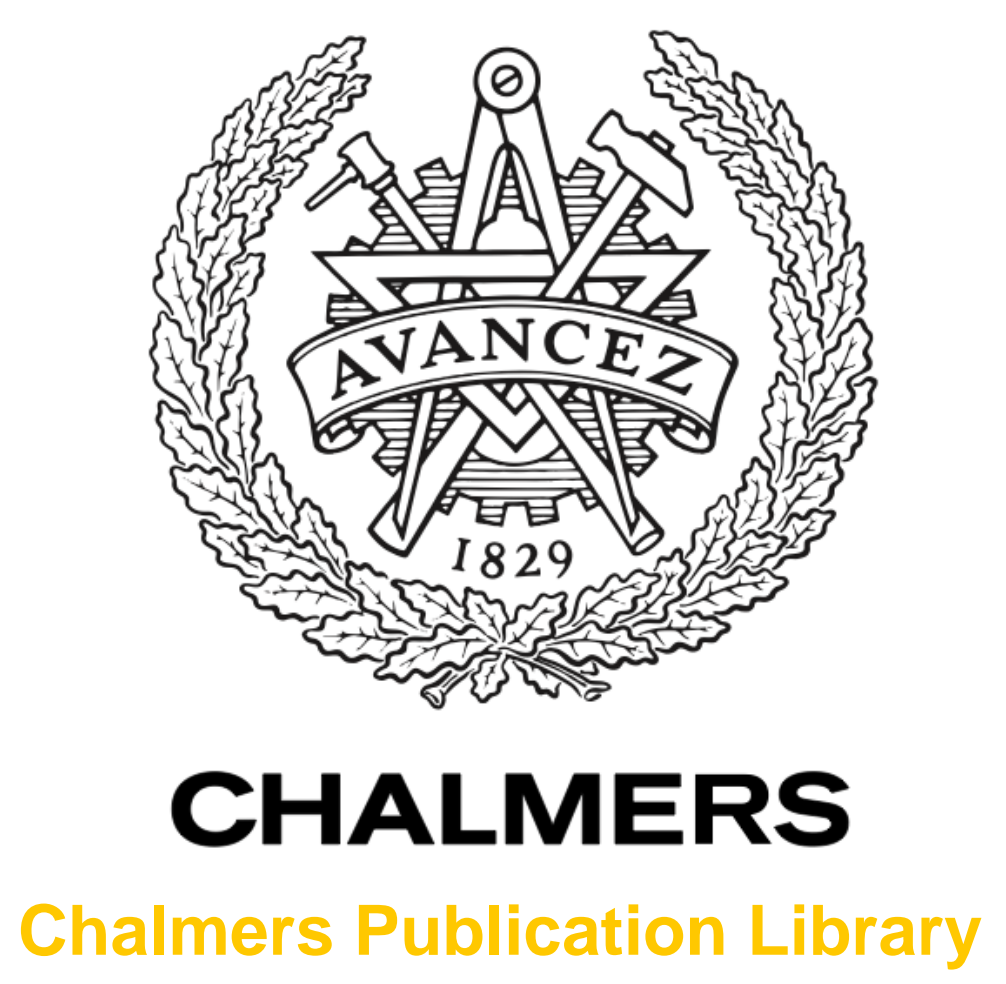

\title{
Numerical studies of bandwidth of parallel-plate cut-off realised by a bed of nails, corrugations and mushroom-type electromagnetic bandgap for use in gap waveguides
}

This document has been downloaded from Chalmers Publication Library $(\mathrm{CPL})$. It is the author's version of a work that was accepted for publication in:

IET Microwaves, Antennas \& Propagation (ISSN: 1751-8725)

Citation for the published paper:

Rajo-Iglesias, E. ; Kildal, P. (2011) "Numerical studies of bandwidth of parallel-plate cut-off realised by a bed of nails, corrugations and mushroom-type electromagnetic bandgap for use in gap waveguides". IET Microwaves, Antennas \& Propagation, vol. 5(3), pp. 282-289.

http://dx.doi.org/10.1049/iet-map.2010.0073

Downloaded from: http://publications.lib.chalmers.se/publication/138299

Notice: Changes introduced as a result of publishing processes such as copy-editing and formatting may not be reflected in this document. For a definitive version of this work, please refer to the published source. Please note that access to the published version might require a subscription. 


\title{
Numerical studies of bandwidth of parallel plate cut-off realized by bed of nails, corrugations and mushroom-type EBG for use in gap waveguides
}

\author{
Eva Rajo-Iglesias, Per-Simon Kildal
}

\begin{abstract}
Recently it has been shown that so-called gap waveguides can be generated in the gap between parallel metal plates. The gap waveguides are formed by metal ridges or strips along which local waves propagate, and normal parallel plate modes are prohibited from propagating by providing one of the surfaces with a texture that generates an artificial magnetic conductor (AMC) or an electromagnetic bandgap (EBG) surface on both sides of the ridges or strips. The bandwidth of the gap waveguide is determined by the cut-off bandwidth of a parallelplate waveguide where one surface has such a texture (and no ridges or strips). The present paper studies the bandwidths (or stop bands) of such parallel-plate cut-offs when the AMC or EBG is realized by a metal pin surface, corrugations, or a mushroom surface. It is shown that cut-off bandwidths of up to $4: 1$ are potentially available, and thereby similar bandwidths should be achievable also for gap waveguides.
\end{abstract}

Index Terms - gap waveguide, bandgap surface, soft and hard surfaces, EBG surface.

\section{INTRODUCTION}

The concept of soft and hard surfaces has been known for a couple of decades [1]. Recently it was demonstrated that the hard surface in the form of corrugations or artificial magnetic conducting strips could be used to prohibit propagation of higher order modes between parallel metal plates and thereby enable improved feeding of radiating slots in one of the plates [2], [3]. By a generalization and extension of the ideas behind these demonstrations a new local gap waveguide was described, being present in the gap between two parallel metal plates if one of these is provided by a texture providing a cutoff of all the normal parallel plate modes, at the same time as a local wave is allowed to propagate in desired directions along metal ridges or strips in the texture [4], [5].

The main performance of the gap waveguide is determined by its ability to create cut-off or stop band for the wave propagation in undesired directions. This is achieved by using a texture in the form of a periodic structure, located around the metal strips or ridges. The periodic structure should emulate a high impedance boundary often referred to as an Artificial Magnetic Conductor, (AMC) as illustrated in Figure 1. The reason is that theoretically a Perfect Magnetic Conducting (PMC) surface located below the surface of a perfect electric conductor (PEC) will create a cut-off of parallel plate modes whenever the spacing between the two plates is smaller than a quarter wavelength. This can readily be seen by studying a plane wave in the gap and trying to satisfy the boundary conditions both at the PMC and the PEC. The PEC above
PMC waveguide can be referred to as an ideal parallel plate cut-off case.

If a narrow metal strip or ridge is added in the AMC or EBG surface, a local wave will be allowed to propagate along it without leaking out in the cut-off region formed by the AMC. This local wave will be a quasi-TEM wave except for the ideal PMC case, when it is a TEM wave.

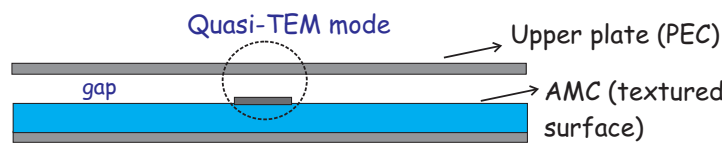

(a) Microstrip

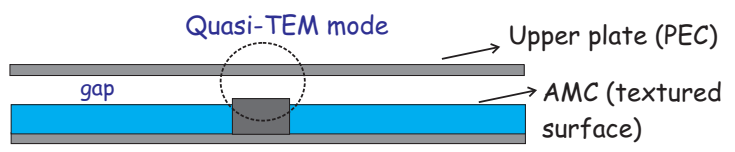

(b) Ridge

Fig. 1. Sketches of the cross-sectional geometry of microstrip- (upper) and ridge-types (lower) gap waveguides, made by metal strip or ridge surrounded by parallel-plate cut-off.

The AMC or EBG surface plays a key role in the design of gap waveguides as the frequency range over which the local quasi-TEM mode can propagate as a single mode is determined by the frequency range over which there is a stop band of the parallel-plate modes. Consequently, an extensive study of the behaviour of different periodic structures (the simpler, the better) in one of the surfaces of a parallel plate waveguide is of interest.

In this sense, this work explores the possibilities of creating parallel-plate cut-offs, i.e. stop bands in parallel plate waveguides, by using different periodic structures, such as a textured surface made of metal pins also referred to as a bed of nails [6], the classical corrugations, and finally the original mushroom-type EBG surface [7]. This will be done by studying the bandgap inside a parallel plate waveguide, or in other words the bandwidth of the parallel plate cut-off, in the same way as the bandgap for surface waves in similar open surfaces were studied in [8].

The metal pin surface was not included in the study in [8], because it is not before recently that it was found to be a very good realization of a magnetic conductor [6]. The pin surface has many advantages because it does not require any dielectric, it is isotropic, and it can be easily manufactured. All this makes it suitable for high frequency designs. Mushroom-type 
EBG surface is of interest for lower frequencies because the mushroom surface can be realized much thinner than the pin surface. Finally, the present study includes also corrugations, even if they are not isotropic, for completeness purposes and to link this work with the previous one [8], where corrugations were the reference surface exhibiting the best performance for surface wave bandgaps. Corrugations show also similar cutoff characteristics as the isotropic AMC surfaces, with the exception that several parallel local waves can propagate, one along each ridge between the corrugations [2]-[3].

The gap waveguides [4]-[5] are results of research on soft and hard surfaces as described in [1]. The ideal soft and hard surfaces can be interpreted as grids of parallel PEC and PMC strips [9]. The gap waveguides consists ideally of a single PEC strip under a PEC plate (above-located ground plane), and on both sides of the PEC strip there is a PMC. Thus, the gap waveguides are formed by a single PEC strip in a PMC plane, opposing a PEC ground plane. The relations to PEC/PMC strip grids are evident.

The results in the present paper are based on computing dispersion diagrams using the Eigenmode Solver of CST Microwave Studio software for several structures and of different dimensions. For simplicity only one direction of propagation is considered coinciding with one of the two principle directions of the periodic lattice, and then afterwards the geometry was checked for wave propagation in the 45 plane. From each dispersion diagram the start and end frequencies of the cut-off band (stop band), within which there are no modes, have been determined. The start and end frequencies of the stop band have thereafter been plotted for each structure as a function of different dimensions. Each change of dimension requires a new calculation of the dispersion diagram.

\section{IDEAL PARALLEL PLATE CUT-OFF REALIZED BY PMC}

We will first study for reference the stop band when the parallel plate waveguide consists of a metal surface and an ideal PMC, separated by a gap $h$. This ideal example is important for understanding the bounds on $h$, of which reason we include it as a reference in several of the graphs to follow. Theoretically, there is one physical limit for the lower (start) frequency of the stop band, and two physical limits for the upper (end) frequency of the stop band. The lower cut-off limit comes from the frequency at which the textured surface starts exhibiting a high enough surface impedance. This lower cutoff can be approximated by the frequency at which the surface impedance change from inductive to capacitive, i.e., a lower capacitive- $Z$ limit. Similarly, there is also an upper cut-off limit at which the surface impedance becomes too small, which can be approximated with the frequency at which it changes from capacitive to inductive again, i.e. an upper capacitive$Z$ limit. However, there is also another second upper limit determined by the gap $h$ itself. Its height must be smaller than $\lambda / 4$ for the ideal PEC above PMC case in order to avoid the propagation of all types of parallel plate modes and not only the dominant TEM mode. This last limit is the only one that determines behaviour for the ideal PEC above PMC parallel plate waveguide. The lower cut-off limit is at zero frequency and the upper impedance-determined limit never appears. The upper cut-off limit for the ideal PEC above PMC case is presented together with simulated results as a function of the gap height $h$ in Figure 2.

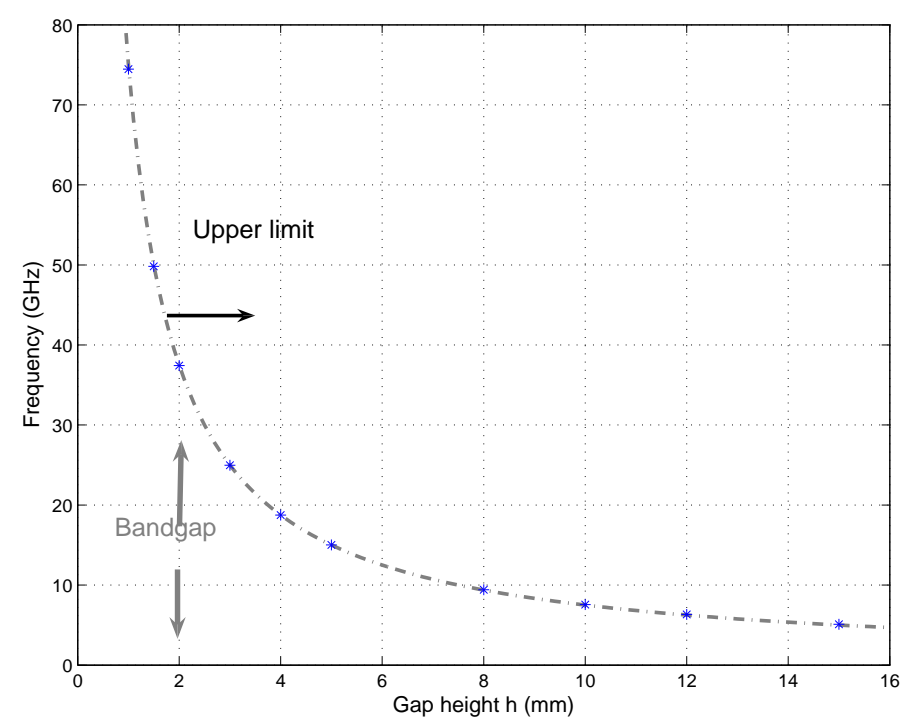

Fig. 2. Analytical (grey line) and computed (*-) end frequency of an ideal parallel plate cut-off realized by PMC.

\section{PARALlEL PLATE CUT-OFF REALIZED B Y BED OF NAILS}

The first case to be studied is the pin surface, also referred to as a bed of nails, the geometry of which is shown in Figure 3. The geometrical parameters that may have effect on the cut-off bandwidth of the parallel plate waveguide are:

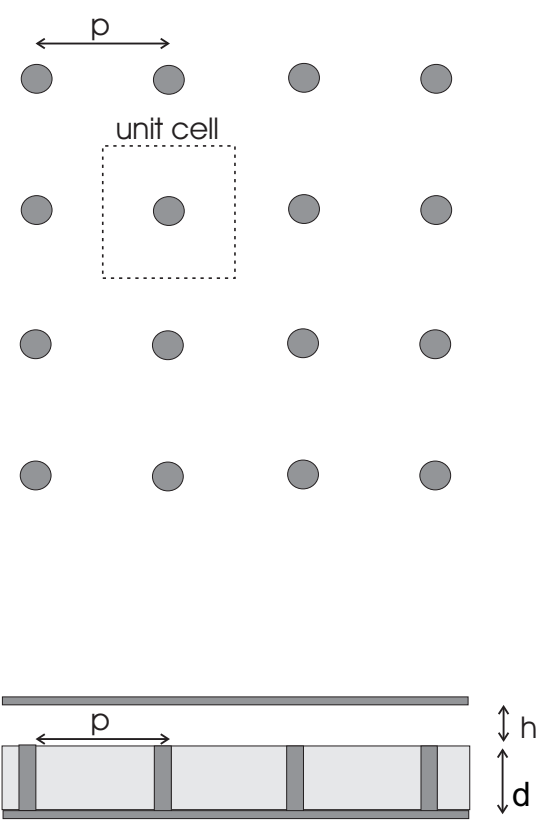

Fig. 3. Geometry of parallel plate waveguide with cut-off realized by a bed of nails in the lower plate.

- The distance to the upper plate, i.e., "the gap height", $h$. This is a parameter which all gap waveguides have 
in common, independently of the type of artificial surface used, and it would be of interest to draw general conclusions about the best value of it.

- The length $d$ of the pins, representing the thickness of the surface layer generating the AMC.

- The period $p$ of the pins.

- The radius $r$ of the pins.

- The geometry of the lattice, i.e., how the pins are regularly disposed (in the example in Figure 3, the lattice is square).

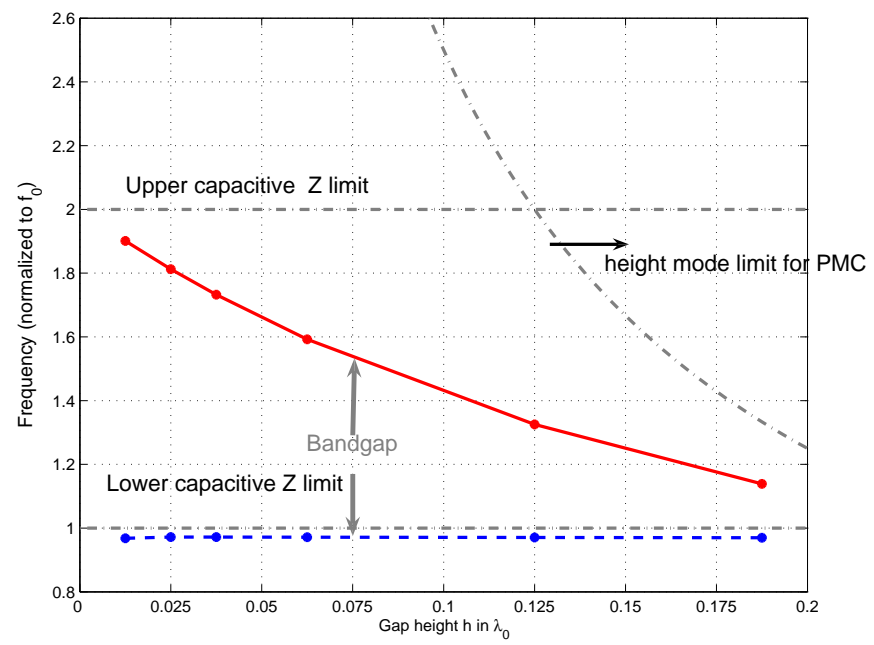

Fig. 4. Computed relative start (dashed line) and end (solid line) frequencies of the stop band of parallel plate waveguide with bed of nails in one plate as shown in Figure 3. The lower and upper capacitive $Z$-limits for the bed of nails are shown as reference, as well as the upper $\lambda / 4$ limit of the cut-off of an ideal PEC above PMC waveguide. The reference $f_{0}$ is the frequency at which the pin length $d=\lambda_{0} / 4$.

As a first result, Figure 4 represents the cut-off limits as a function of the gap height $h$ for an example where $d=20 \mathrm{~mm}$, $p=3 \mathrm{~mm}$ and $r=0.2 \mathrm{~mm}$. The bandgap is defined by a start frequency at which the wave propagation is stopped and an end frequency at which waves can propagate again, represented by a dashed blue line and solid red line, respectively. As already mentioned, there is a lower limit for the start frequency of the stop band and two upper limits for the end frequency. In Figure 4 we have plotted these three limits as grey "dot-dash" lines. The lower capacitive- $Z$ limit for the start frequency is approximated by using the rules that apply for corrugations, i.e. grooves (pins in our case) need to be at least $d=\lambda / 4$ deep. We see that this rule applies approximately for the pins as well, because the plotted lower limit is very close to the actual computed start frequency. The corresponding upper capacitive- $Z$ limit of the end frequency of the stop band appears approximately when $\mathrm{d}=\lambda / 2$, also by analogy with corrugations.

From this first example it is already clear how the gap $h$ is a key parameter which strongly affects the size of the stop band. This motivates the study of the effect of this parameter, when other parameters of the structure are also varied, such as the period of the pins $p$. The two graphs in Figure 5 contain the effect of the gap size $h$ on the start and end frequencies of the

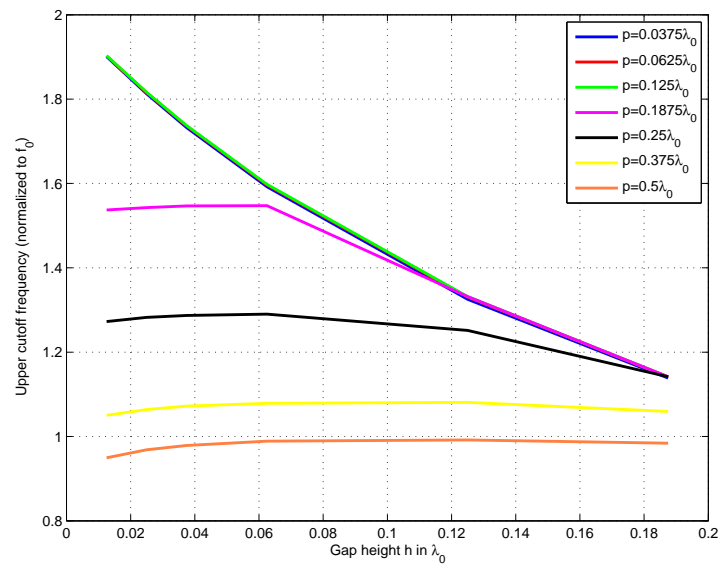

(a) End frequency

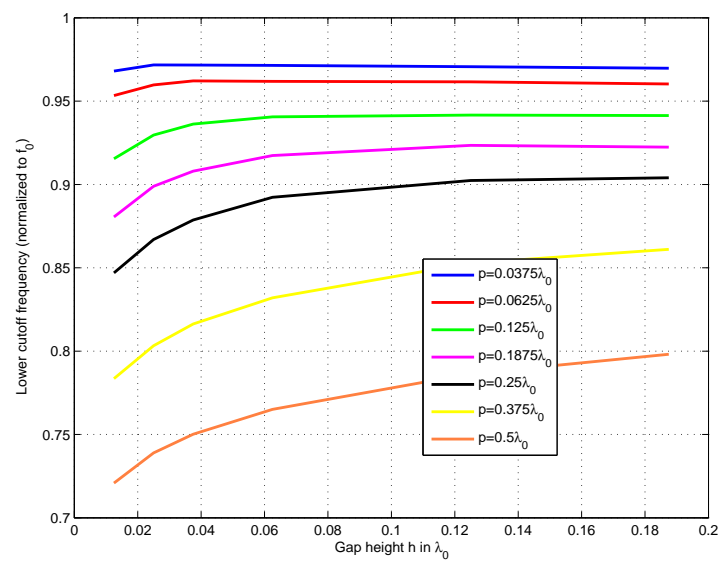

(b) Start frequency

Fig. 5. Start (a) and end (b) frequencies of the stop band as a function of gap height $h$ for different pin periods $p$, for the parallel plate waveguide with bed of nails in Figure 3. The pins are circular with length $d=0.25 \lambda_{0}$ and radius $r=0.0025 \lambda_{0}$.

stop band, respectively. The frequency used for normalization in the graphs is the frequency $f_{0}=3.75 \mathrm{GHz}$ at which the pins height $d=\lambda_{0} / 4$. The height $d$ and radius $r$ of the pins are kept the same as in the initial example. We see that for all periods the size of the stop band increases when the height $h$ of the gap to the upper plate decreases.

The start frequency of the stop band reduces when the period of the pins increases due to some increase of the effective electrical length of the pins. On the other hand, the end frequency is almost unaffected by the period as far as this is small enough. When the period becomes larger, the upper limit is dramatically reduced due to the propagation of new modes. Moreover, for periods larger than $0.25 \lambda_{0}$ the end frequency of the stop band is almost not changing at all with the gap size $h$, and the increase of the stop band is entirely due to the change in the start frequency, which goes significantly down for small gap heights.

The total relative bandwidth $\left(f_{\text {end }} / f_{\text {start }}\right)$ is summarized in Figure 6 as a function of period and gap height. Starting from the smallest period, this bandwidth increases with period for all gap sizes, up to a certain height-dependent period, corresponding to where a new mode appears in the dispersion 
diagram. The frequency at which this periodicity mode appears depends much more slowly on the gap height than the mode appearing when the gap height becomes larger. Therefore, the latter height mode limits the stop band for larger heights rather than the periodicity mode. We can conclude from these results that when a large bandwidth is required, small periods as well as small heights have to be used.

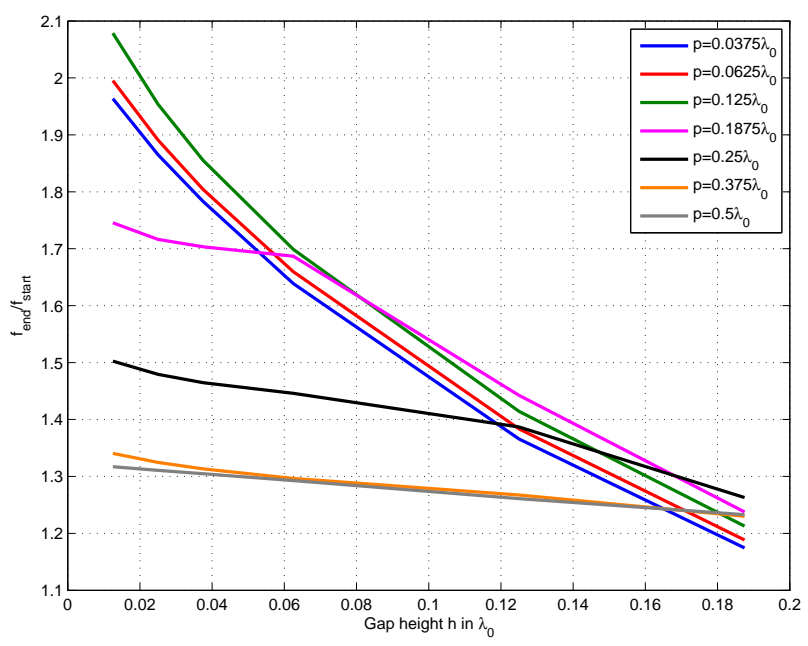

Fig. 6. Relative cut-off bandwidth $\left(f_{\text {end }} / f_{\text {start }}\right)$ as a function of the gap heights $h$ for a parallel plate waveguide with cut-off realized by a bed of nails for different periods of the pins $p$. The pins are circular with length $d=0.25 \lambda_{0}$ and radius $r=0.0025 \lambda_{0}$.

The radius of the pins $r$ is now considered. This parameter has been changed while keeping a constant period $p$. The ratio between radius and period $r / p$ has been chosen as the parameter to be represented in the horizontal axis of the following figures summarizing the simulation results. Initially, a period of $20 \mathrm{~mm}$, i.e., $0.25 \lambda_{0}$, was selected. The results corresponding to this case are represented in Figure 7 for three different upper plate heights $h$. As in the previous study concerning the period, the effect of the gap height $h$ is a reduction in the size of the stop band when this height increases. The start and end frequencies of the stop band move in opposite directions when the pin radius over period increases reaching a maximum when the ratio is between 0.05 and 0.2, depending on the gap height. Figure 7 shows results for square pins, but circular pins show very similar results, as compared for a specific case $(h=5 \mathrm{~mm}, p=20 \mathrm{~mm}$ and $d=20 \mathrm{~mm}$ ) in Figure 8. For analogy we keep the word "radius" to refer to half the width of the square pins.

Related to the same parameter, new results were calculated for the case where the period is changed. In fact, to modify the $r / p$ keeping the period constant means to reduce the physical space between pins which in the end means, for a given period, (especially for a large one) to avoid the propagation of higher order modes between pins. This motivates the study of this parameter $r / p$ by changing the period $p$. In this occasion the gap height was selected as $h=5 \mathrm{~mm}$, i.e., $0.0625 \lambda_{0}$. The results are included in Figure 9. The qualitative behaviour is similar to the one in the precedent studies. It is noted that a larger period moves the start frequency of the stop band

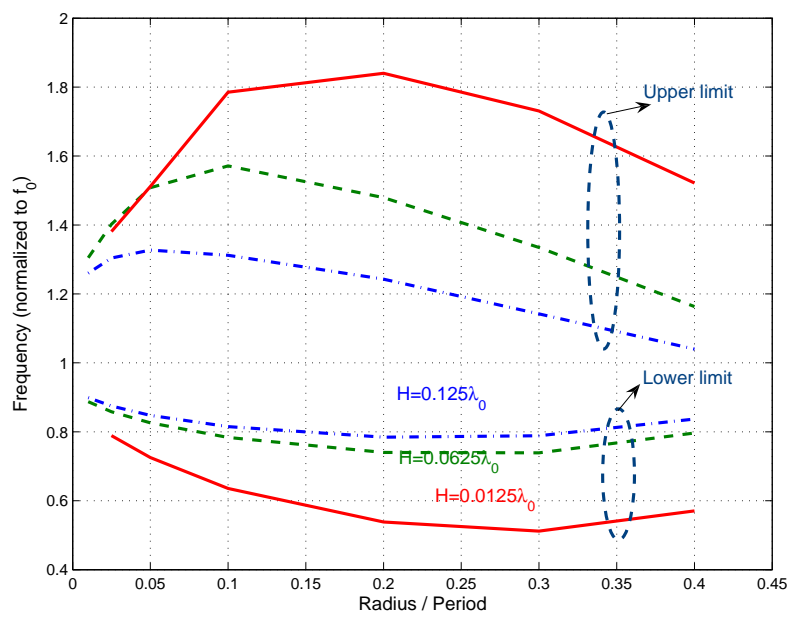

Fig. 7. Normalized start and end frequencies of the stop band of the parallel plate waveguide with cut-off realized by a bed of nails showing effect of the radius/period for different gap heights $h$. The pins are square with length $d=0.25 \lambda_{0}$ and radius $r=0.0025 \lambda_{0}$.

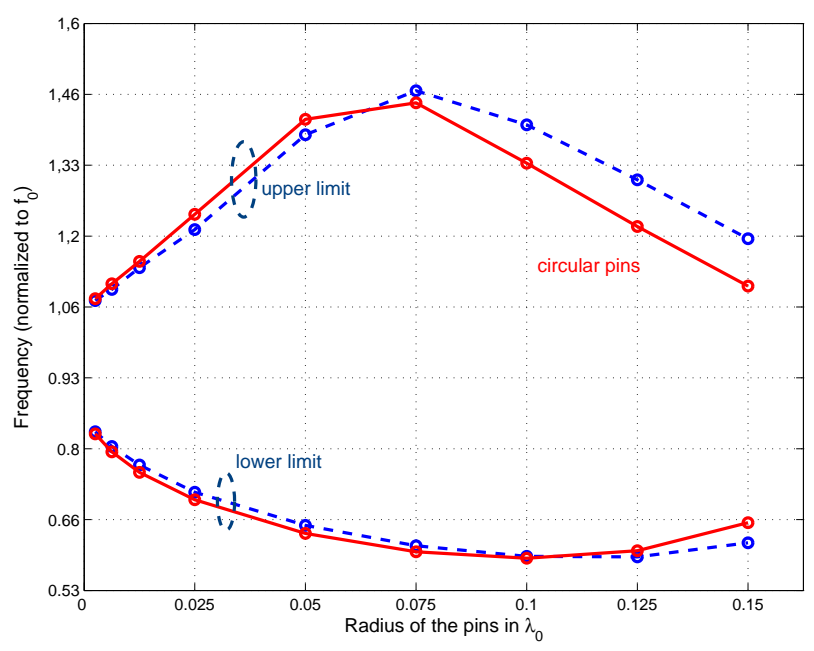

Fig. 8. Normalized start and end frequencies of the stop band of the parallel plate waveguide with cut-off realized by a bed of nails showing effect of square versus circular pins for pin length $d=0.25 \lambda_{0}$, gap height $h=$ $0.0625 \lambda_{0}$ and period $p=0.25 \lambda_{0}$.

towards lower frequencies but also affects the end frequency, particularly when the ratio between radius and period $r / p$ is small. As a consequence, the largest stop band for small gaps is obtained for small periods. The relative bandwidth $\left(f_{\text {end }} / f_{\text {start }}\right)$ has also been computed for this parameter and it is represented in Figure 10. Only when the radius is much smaller than the period, the structure with smaller period exhibits the largest relative bandwidth. When the radius is relatively thick, the structures with larger periods achieve larger bandwidths.

As last parameter for this geometry, the lattice has been modified. From photonics [10], we know that the effect of this parameter in the stop band cannot be neglected. For simplicity, only the triangular lattice replacing the squared one has been analysed. A summary of this analysis is illustrated in Figure 11 that compares both the effect of the gap height and the radius of the pins for the two types of lattices. Even if the 


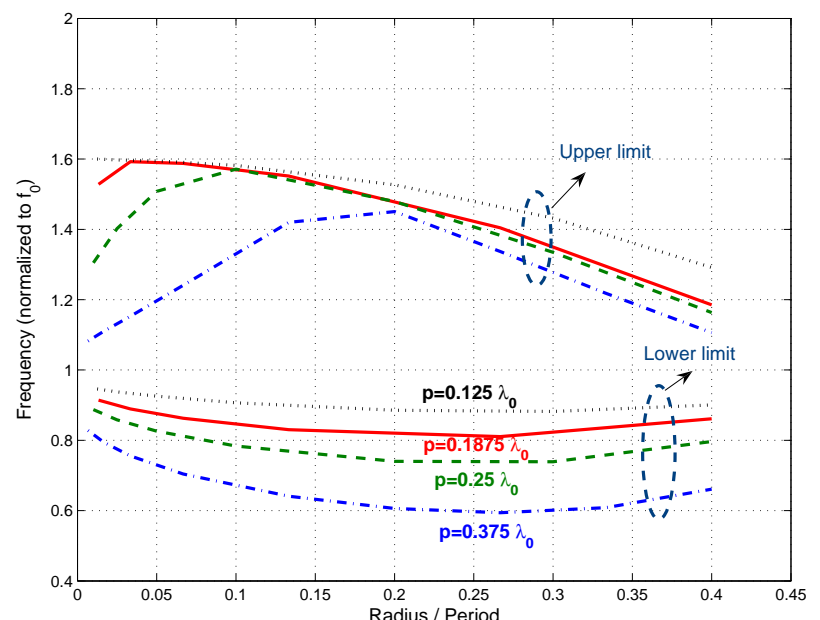

Fig. 9. Normalized start and end frequencies of the stop band of the parallel plate waveguide with cut-off realized by a bed of nails showing effect of the radius/period for different periods $p$. The pins are square with length $d=0.25 \lambda_{0}$. The gap height is $h=0.0625 \lambda_{0}$.

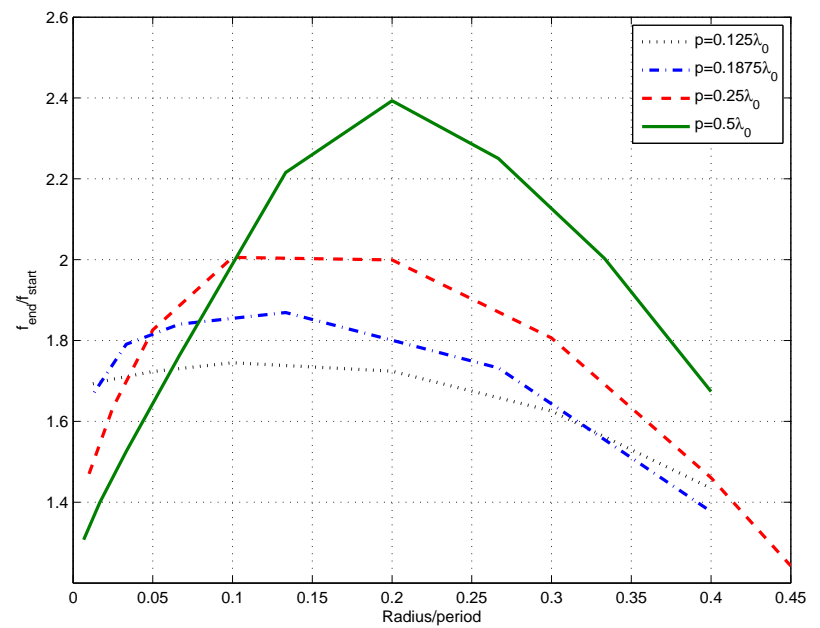

Fig. 10. Relative cut-off bandwidth of a parallel plate waveguide with cut-off realized by a bed of nails as a function of the radius/period of the pins for different periods $p$. The pins are square with length $d=0.25 \lambda_{0}$ and the gap height is $h=0.0625 \lambda_{0}$.

results are not identical, they are similar enough to consider this parameter as a secondary one in defining the stop band properties of this structure, at least when comparing it to the other studied parameters.

\section{PARALlEL PLATE CUT-OFF REALIZED BY CORRUGATIONS}

The classical high impedance surface is the corrugated surface. This surface offers an anisotropic behaviour, which means that the high impedance condition is appearing only for one polarization and for one propagation direction. This open surface (without a PEC above it) has been previously studied as a bandgap surface for surface waves in [8] and it exhibited the largest bandgap for surface waves. The surface anisotropy does not add any direct advantage for application in gap waveguides except that it will appear when several parallel gap waveguides are used e.g. to feed a slot array [2]. Therefore, we

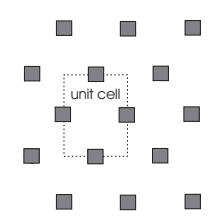

(a) Triangular lattice

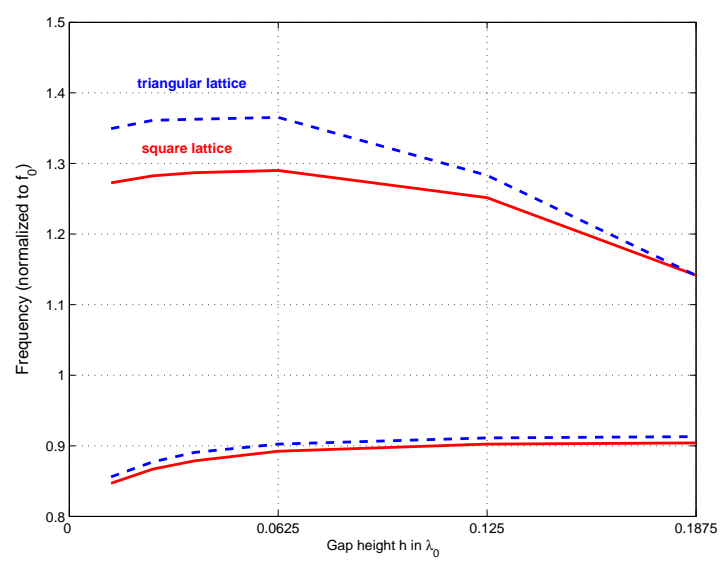

(b) Effect of gap $h$

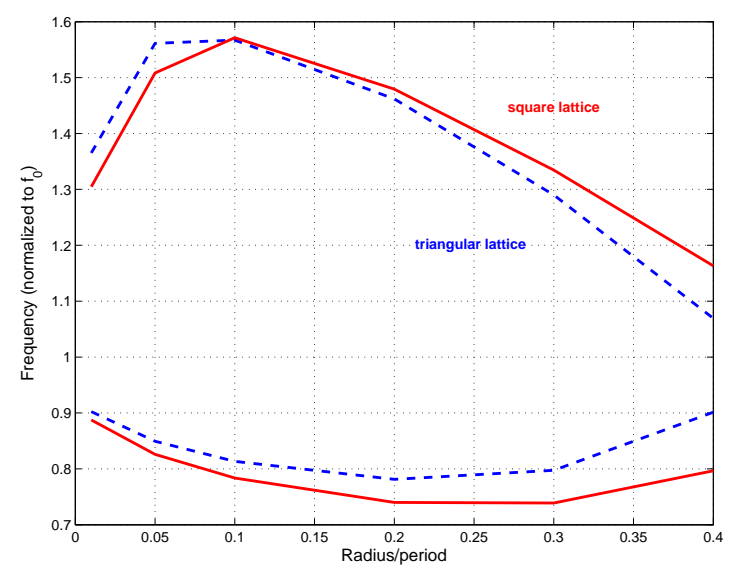

(c) Effect of radius $r$

Fig. 11. Comparison of normalized start and end frequencies of the stop band for a parallel plate waveguide with cut-off realized by metal pins located in rectangular and triangular lattices.

include a parametric study of this classical surface when it is covered by an upper metal plate. The particular parameters for tuning this surface are the period $p$ of the corrugations and the ratio between the groove and metal widths $a / p$ (described in Figure 12), assuming that, as for the pin's case, the depth of the corrugations is approximately $0.25 \lambda_{0}$ at the desired frequency. The combined effect of the latter parameters with the gap size $h$ is studied in this section. The dispersion diagrams are in this case computed only in the "soft" direction transverse to the corrugations.

First, the period, $p$ was varied from $0.0625 \lambda_{0}$ to $0.25 \lambda_{0}$ while keeping $a / p=0.25$ constant. The end frequency of the stop band is insensitive to this parameter (seen in Figure 13), whereas the start frequency moves down in frequency when the corrugation period increases, similar to the bandgap in [8]. The effect of varying the gap height $h$ appears similar to the 
variation of the gap height for the bed of nails case. Small gaps provide the largest stop band, in this example up to $3: 1$ bandwidths.
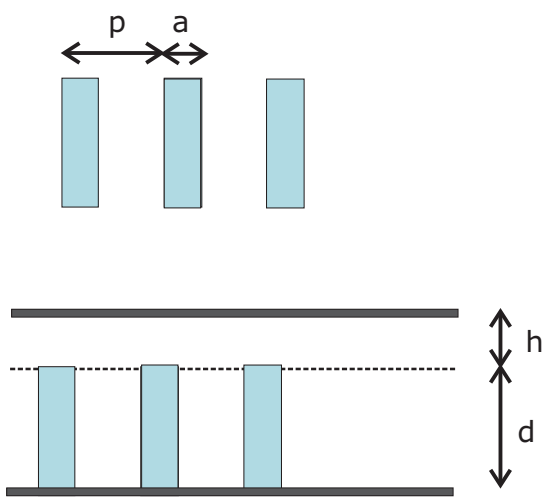

Fig. 12. Geometry of studied parallel-plate waveguide with cut-off realized by corrugations.

When the period is fixed to $\lambda_{0} / 8$ and the $a / p$ parameter is modified, we get the results presented in Figure 14. Now, the end frequency of the stop band moves up in frequency when $a / p$ decreases, consequently, the stop band size increases. When we studied bandgaps of surface waves in [8] we found that the best performance was obtained when both the period and the $a / p$ approach zero. The same conclusion can be derived for the parallel plate case when the corrugated surface is topped by a metal upper plate.

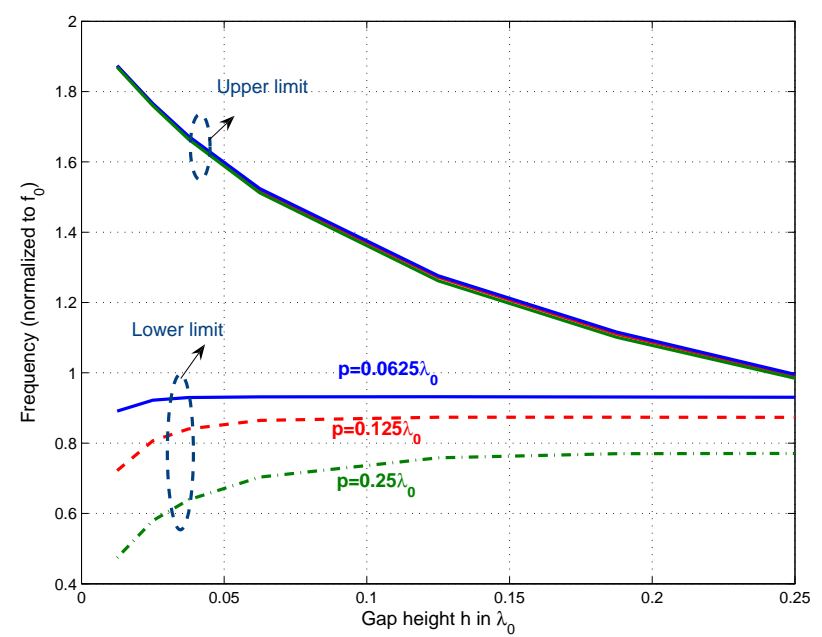

Fig. 13. Normalized start and end frequencies of stop band of parallel plate waveguide with cut-off realized by corrugations showing effect of period $p$ as a function of gap height $h$ for $a / p=0.25$.

\section{PARAllel Plate CUt-off Realized By MUSHROOM-TYPE EBG}

Finally, the original mushroom-type EBG is studied under the same premises. For analogy with the previous surfaces, no dielectric material was considered. Obviously, the manufacturing of this structure would in general, require a substrate material supporting the printed patches. For this reason, and

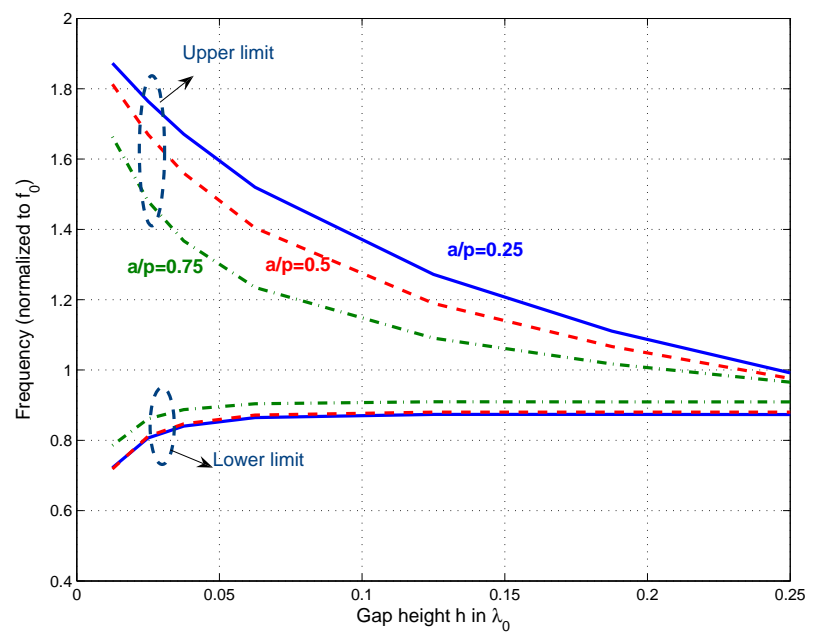

Fig. 14. Normalized start and end frequencies of stop band of parallel plate waveguide with cut-off realized by corrugations showing effect of $a / p$ as a function of gap height $h$ for $p=\lambda_{0} / 8$.
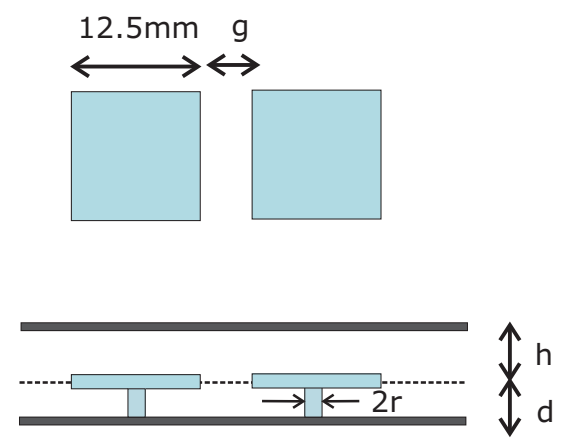

Fig. 15. Geometry of studied parallel-plate waveguide with cut-off realized by mushroom-type EBG.

also because of the reduced unit cell volume, this geometry is more appropriated for use at lower frequencies.

In this case it is difficult to define a frequency directly related to any dimension of the geometry that can be used for normalization purposes, like we did for the previous geometries. Therefore, in this last case the results will be presented without normalization and the side of the patch will be arbitrarily taken as $12.5 \mathrm{~mm}$.

Among the parameters of the structure, we initially analyze the consequences of modifying the gap $g$ between contiguous mushrooms, see Figure 15. It is noted that the effect of $g$ on the stop band is not very significant, except for the case the gap height $h$ is small, as shown in Figure 16.

The radius of the grounded pin is another of the parameters to be taken into account. Its effect on the stop band is presented in Figure 17. Mainly, both limits of the stop band move in the same direction when the radius changes, thus, it is not directly affecting the stop band size but its position in the spectrum. The larger the radius, the higher the frequency. This can be understood by remembering that the resonance in this structure is happening in the loop created between neighboring mushrooms and increasing the radius is equivalent to reducing that loop.

The last parameter to be considered is the thickness of 


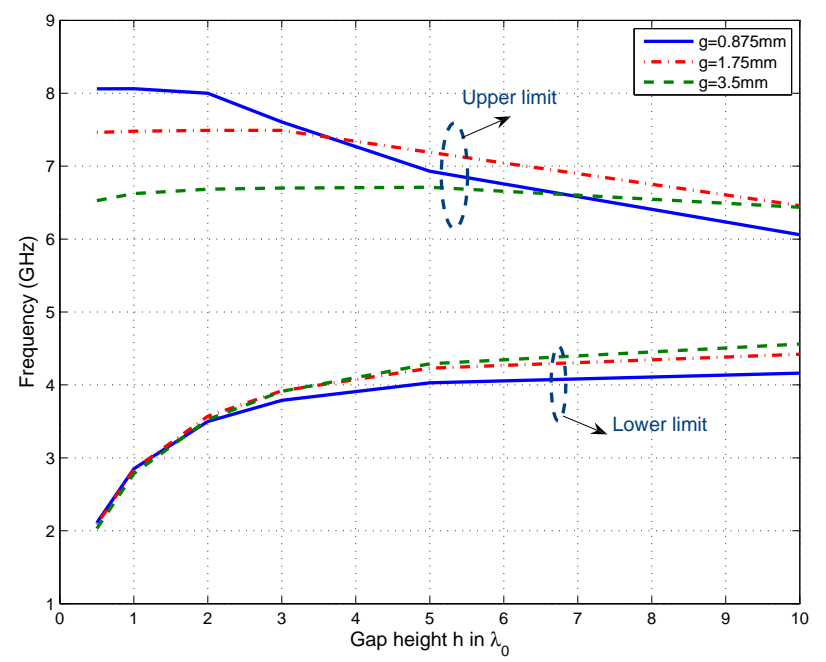

Fig. 16. Start and end frequencies of stop band of parallel plate waveguide with cut-off realized by mushroom-type EBG surface showing effect of gap parameter $g$ as a function of gap height $h$. The mushroom surface has dimensions $d=3 \mathrm{~mm}$ and $r=1 \mathrm{~mm}$.

the substrate $d$ or the distance from the patch to the ground plane. Figure 18 summarizes the consequences of a change in this parameter. The start frequency is completely shifted in frequency depending on the value of this thickness, the thicker the substrate, the lower the frequency and this happens independently of the distance to the upper plane $h$. This behaviour coincides with the one achieved for bandgaps of surface waves in the open EBG mushroom surface [8]. On the other hand, the end frequency is almost unaffected by this parameter when the gap to the upper plate $h$ is small, but follows the variation of the start frequency for medium to large values of such gap.

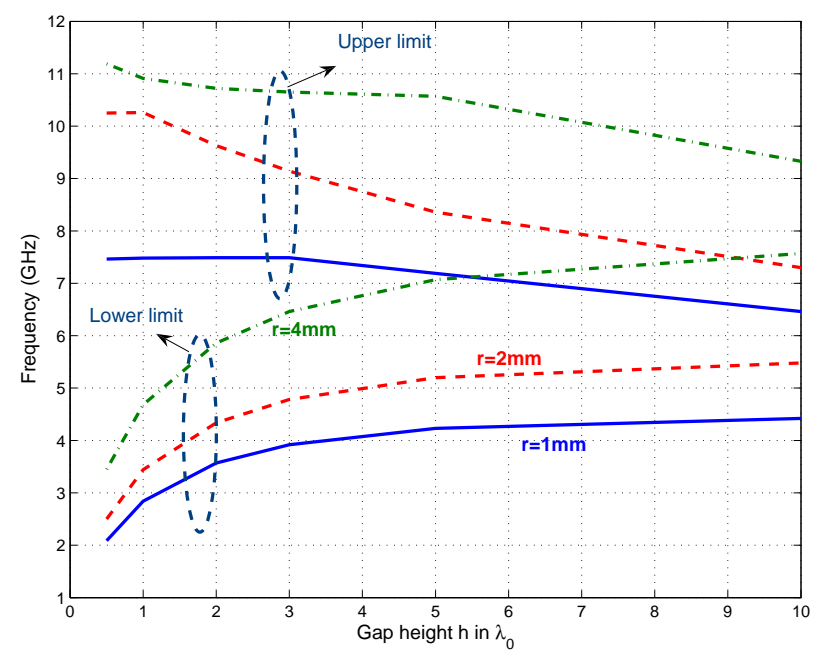

Fig. 17. Start and end frequencies of stop band of parallel plate waveguide with cut-off realized by mushroom-type EBG surface showing effect of the radius $r$ as a function of gap height $h$. The mushroom surface has dimensions $d=3 \mathrm{~mm}$ and $g=1.75 \mathrm{~mm}$.

Among the presented results, it is possible to find bandwidths approaching 4:1.

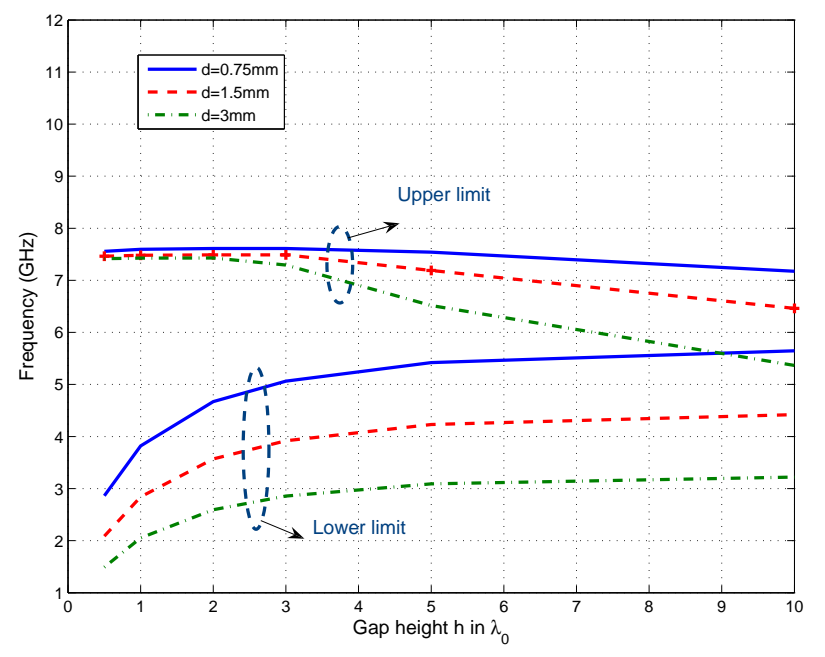

Fig. 18. Start and end frequencies of stop band of parallel plate waveguide with cut-off realized by mushroom-type EBG surface showing effect of the thickness $d$ of mushroom surface as a function of gap height $h$. The mushroom surface has dimensions $g=1.75 \mathrm{~mm}$ and $r=1 \mathrm{~mm}$.

\section{CONCLUSION}

Parallel plate gap waveguides appear as a new promising transmission line technology for millimeter waves. Its conceptual design involves the use of an artificial surface to stop wave propagation in undesired directions, i.e. to generate parallel plate cut-off in undesired direction. The present paper has studied the stop band for three different ways of realizing the cut-off; by a bed of nails, by transverse corrugations and by mushrooms, i.e. metal patches with vias. It is assumed that all three surfaces are entirely made of metal, in order to keep the losses as small as possible.

A complete parametric study of the cut-off bandwidths of these surfaces has been performed. The result is that octave cut-off bandwidths are available using bed of nails, and two octaves when using mushroom-type EBG surfaces. Corrugations have similar cut-off characteristics as the pin surface, but only in the transverse direction. They will guide local gap waves along each ridge ([2] and [11]), and the cut-off characteristics in the transverse direction define how well normal global parallel plate modes are suppressed. The presented graphs can be used to select dimensions of pins, corrugations and mushrooms that fit to the desired center frequency, bandwidth, size requirements and manufacture method of the considered application.

\section{ACKNOWLEDGEMENT:}

This work has been supported in part by The Swedish Foundation for Strategic Research (SSF) within the Strategic Research Center Charmant and also by the Spanish government by Jose Castillejo mobility grant program and under project CONSOLIDER-INGENIO CSD2008-00068 (TERASENSE).

\section{REFERENCES}

[1] P.-S. Kildal, "Artificially soft and hard surfaces in electromagnetics," IEEE Transaction on Antennas and Propagation, vol. 28, no. 10, pp. 1537-1544, 1990. 
[2] A. Valero-Nogueira, E. Alfonso, J. Herranz, and M. Baquero, "Planar slot-array antenna fed by an oversized quasi-TEM waveguide," $\mathrm{Mi}$ crowave and Optical Technology Letters, vol. 49, pp. 1875-1877, August 2007.

[3] P. P. de la Torre, J. Fernández, and M. S.-C. ner, "Characterization of artificial magnetic conductor strips for parallel plate planar antennas," Microwave and Optical Technology Letters, vol. 50, pp. 498-504, February 2008.

[4] P.-S. Kildal, "Waveguides and transmission lines in gaps between parallel conducting surfaces." European Patent Application EP08159791.6, 7 July 2008.

[5] P.-S. Kildal, E. Alfonso, A. Valero-Nogueira, and E. Rajo-Iglesias, "Local metamaterial-based waveguides in gaps between parallel metal plates," IEEE Antennas and Wireless Propagation Letters, vol. 8, pp. 8487, 2009.

[6] M. Silveirinha, C. Fernandes, and J. Costa, "Electromagnetic characterization of textured surfaces formed by metallic pins," Antennas and Propagation, IEEE Transactions on, vol. 56, Feb. 2008.

[7] D. Sievenpiper, L. Zhang, F. Jimenez-Broas, N. Alexopolous, and E. Yablonovitch, "High-impedance electromagnetic surfaces with a forbidden frequency band," IEEE Trans. Microwave Theory and Techniques, vol. 47, pp. 2059-2074, November 1999.

[8] E. Rajo-Iglesias, M. Caiazzo, L. Inclán-Sánchez, and P.-S. Kildal, "Comparison of bandgaps of mushroom-type EBG surface and corrugated and strip-type soft surfaces," IET Microwaves, Antennas and Propagation, vol. 1, pp. 184-189, February 2007.

[9] P.-S. Kildal and A. Kishk, "EM modeling of surfaces with STOP or GO characteristics. Artificial magnetic conductors and soft and hard surfaces," Applied Computational Electromagnetics Society Journal, vol. 18 , no. 1 , pp. 32-40, 2003.

[10] J. Joannopoulos, R. Meade, and J. Winn, Photonic Crystals: Molding the Flow of Light. Princeton University Press, 1995.

[11] E. Alfonso, P.-S. Kildal, A. Valero, and J. Herranz, "Detection of local quasi-tem waves in oversized waveguides with one hard wall for killing higher order global modes," IEEE International Symposium on Antennas and Propagation (IEEE AP-S), San Diego, July 2008. 